\title{
Excessive Fear/ Psychological Threat of Getting Covid-19 Infection in People Can Lead to Accidental Foreign Bodies in Nose During Screening and Sampling
}

\author{
Palvi Mahajan ${ }^{1}$ and Satinder Pal Singh ${ }^{2 *}$ \\ ${ }^{1}$ Junior Resident, Department of Otolaryngology \& Head Neck Surgery, Government Medical College, India
}

${ }^{2}$ Senior Resident, Department of Otolaryngology \& Head Neck Surgery, Government Medical College, India

*Corresponding author: Satinder Pal Singh, Senior Resident, Department of Otolaryngology \& Head Neck Surgery, Government Medical College, India

\begin{abstract}
Background: Coronavirus disease 2019 (COVID-19) is an infectious disease caused by severe acute respiratory syndrome coronavirus 2 (SARS-CoV-2).The cases of Covid 19 are increasing daily since last 8 to 9 months due to which testing has also been increased and the incidence of accidental foreign body (swab stick) in the nose and mouth has also been seen during screening and sampling.

Case Report: This paper shows complication of viral swab testing during sampling of suspected irritable, anxious, and stressed Covid 19 patients as swab stick breaks at the indentation point while taking Nasopharyngeal and Oropharyngeal samples resulting in impaction in the nasal cavity or mouth.

Conclusion: This paper highlights various ideas to reduce the chances of accidental foreign body in the nasal cavity and mouth during sampling of suspected covid 19 patients.

Keywords: Covid-19; psychological threat; nasopharyngeal sampling; foreign bodies

Abbreviations: NP: Nasopharyngeal; OP: Oropharyngeal; FB: Foreign Body; ENT: Ear Nose Throat; PPE- Personal Protective Equipment's
\end{abstract}

\section{Introduction}

In Otorhinolaryngology, foreign body in the ear, nose, throat is very frequently seen. Nasal foreign bodies (NFBs) are a common complaint in the emergency department typically in young children. Less commonly patients may be older children, adolescents, or adults with intellectual disabilities [1]. These patients are usually anxious, fearful, irritable and have low mood sometimes they are suffering from depression also.

SARS-CoV-2, the novel coronavirus responsible for COVID-19, first emerged in late 2019 in China. Over the past several months, infection has spread throughout the globe, resulting in a pandemic declared by the World Health Organization (WHO) on March 11, 2020 [2]. During covid 19 pandemic, nasopharyngeal and oropharyngeal samples are being taken of suspected patients and positive patients by ENT department. Sometimes during sample taking patient's reluctant behaviour leads to breakage of swab stick from the indentation point inside the nasal or oral cavity which presents as an unusual foreign body in the nose or mouth. It has become a complication of the sample taking in covid 19 patients and has also become burden and a risk factor the ENT Residents. Here we want to discuss the case of unusual nasal foreign body in a patient of covid 19 during mass sampling. 


\section{Method of Sampling for Covid-19}

Techniques of taking nasopharyngeal and oropharyngeal swab samples in Covid 19 patients [3]

\section{Nasopharyngeal (NP) swab/Oropharyngeal (OP) (Thro- at) swab}

Use synthetic fiber swabs with plastic or wire shafts . Both NP and OP swabs are collected, they should be combined in a single tube to maximize test sensitivity and limit use of testing resources.

NP Swab: Insert minitip swab with a flexible shaft (wire or plastic) through the nostril parallel to the palate (not upwards) until resistance is encountered or the distance is equivalent to that from the ear to the nostril of the patient, indicating contact with the nasopharynx (Figure 1). Swab should reach depth equal to distance from nostrils to outer opening of the ear. Gently rub and roll the swab. Leave swab in place for several seconds to absorb secretions. Slowly remove swab while rotating it. Specimens can be collected from both sides using the same swab, but it is not necessary to collect specimens from both sides if the minitip is saturated with fluid from the first collection. If a deviated septum or blockage create difficulty in obtaining the specimen from one nostril, use the same swab to obtain the specimen from the other nostril.

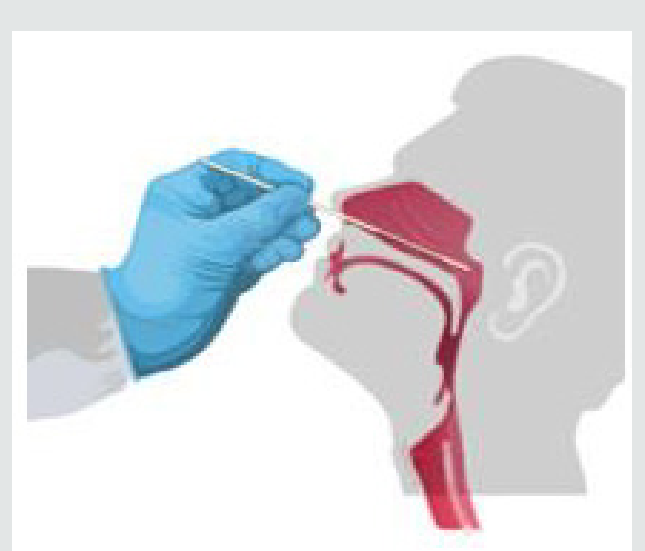

Figure 1: Figure showing technique of taking Nasopharyngeal Swab.

OP Swab: Insert swab into the posterior pharynx and tonsillar areas. Rub swab over both tonsillar pillars and posterior oropharynx and avoid touching the tongue, teeth, and gums.

\section{Case Report 1}

A 38-year-old male patient presented to flu corner of Government medical college Amritsar, Punjab, North India with symptoms resembling that of Covid 19 who was shifted to corona suspect ward for isolating him as well as for taking his NP and OP samples. Patient was anxious, irritable and had low mood. Patient was being counselled by psychiatrist for his neuropsychiatric symptoms to relieve his anxiety and to reduce his fear regarding covid infection and was motivated so that he can give his NP and OP samples. An ENT Resident was being called to take his both samples. After wearing PPE and taking all the precautionary measures, patient was told about the whole procedure of sample taking and was advised to stay calm and to not resist while swab is put inside the nasal and oral cavity. But patient was seemed frightened and fretful (Figure 2). Patient was made to lie down in proper position ,swab stick was inserted into one nasal cavity (Figures $3 \& 4$ ) but patient resisted due which swab stick got broken from the indentation point and got stuck inside the nasal cavity. Patient was again counselled to calm him and anxiolytic drugs were given to reduce his apprehension .After sometime patient was again made to lie down and nasal cavity was inspected and foreign body (swab stick) was localized as one end of the FB can be easily seen in the anterior part of the nasal cavity (Figures 5 \& 6). Under light source, FB was removed with the help of Tilley forceps. Nasal cavity was again visualized for any trauma. Posterior pharyngeal wall also inspected. Xylometazoline nasal drops were put in the nasal cavity prophylactically. Then again with new swab sticks, NP and OP samples were taken and kept in universal viral transport media which was closed and sealed with parafilm and kept in secondary container (falcon tube) and sealed again with parafilm and stored at 4 degree Celsius.

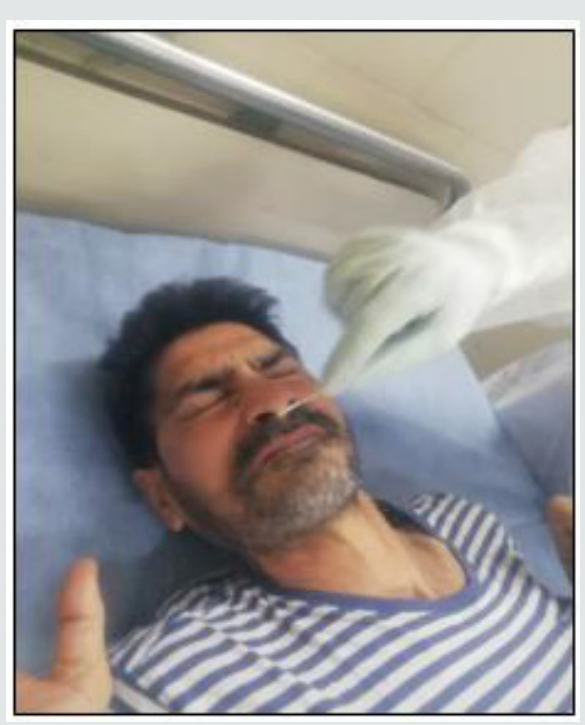

Figure 2: Figure shows insertion of swab stick into nasal cavity of patient who seems to be fearful and irritable. 

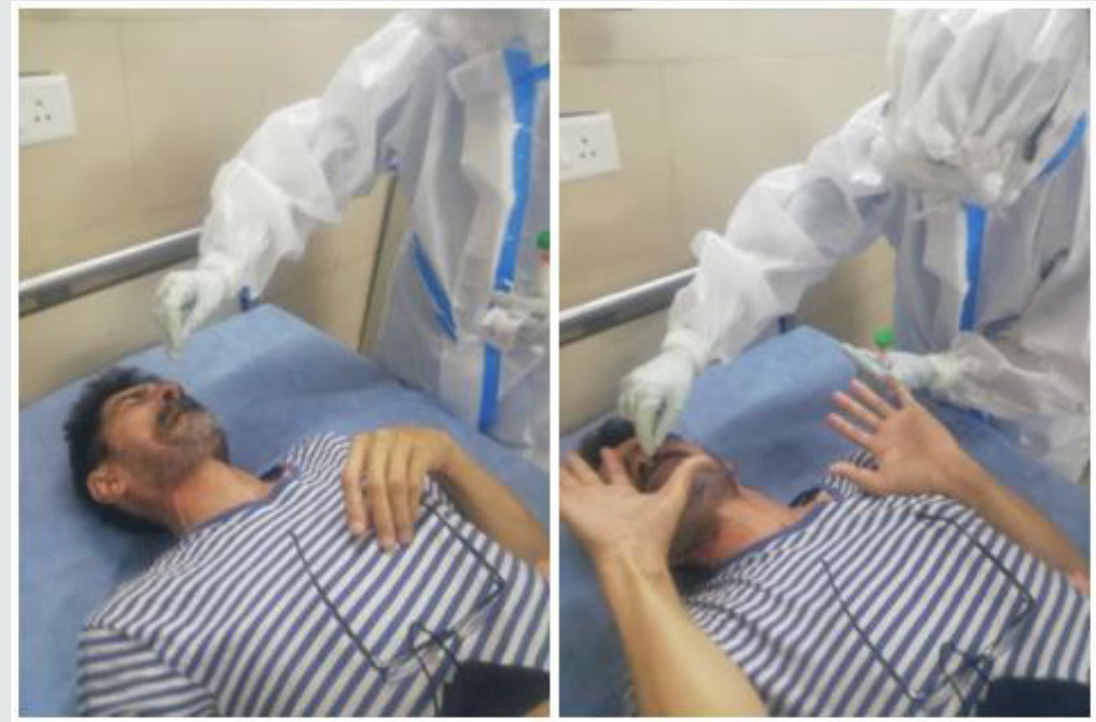

Figure 3 \& 4: Figure shows patient is irritable and resisting insertion of swab stick into the nasal cavity.
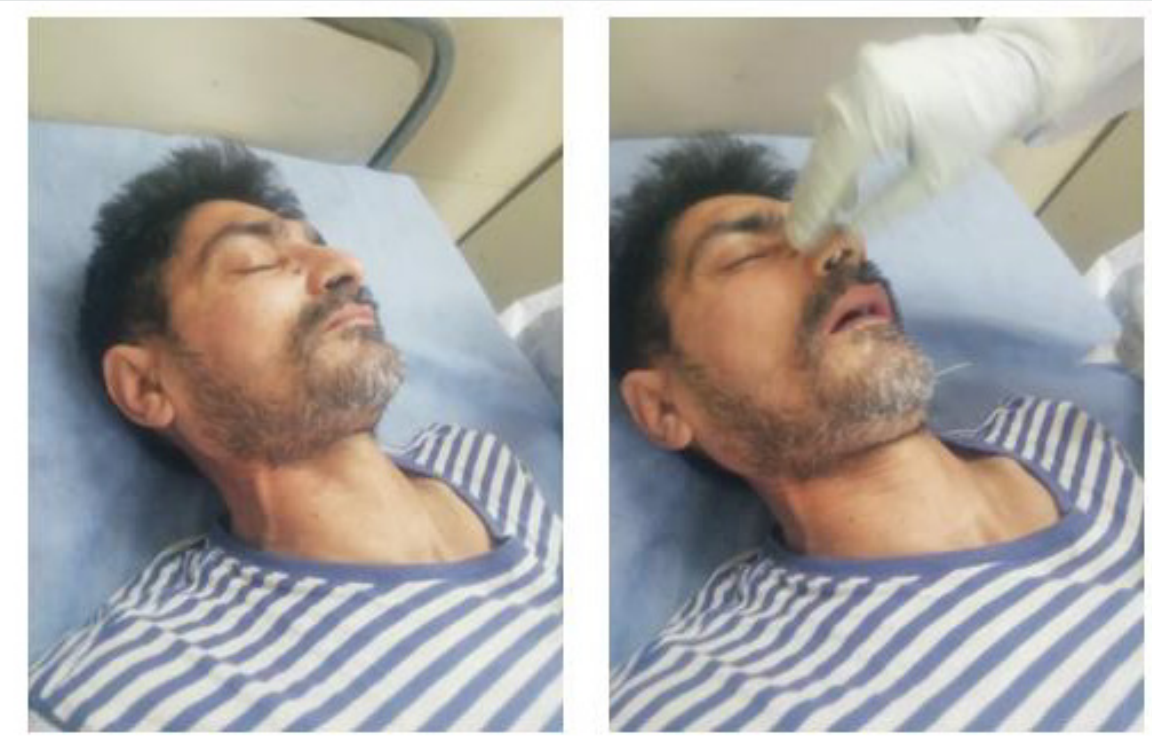

Figure 5 \& 6: Figure shows swab stick (FB) in the anterior part of nasal cavity.

\section{Case Report -2}

A 26-year-old mentally retarded female presented at flu corner of Government medical college, Amritsar with complain of fever and cough who was being shifted to suspect ward of covid 19. ENT Resident was being called for taking her NP and OP swab samples. aal the protective gears were worn and then patient was inspected. Patient was not understanding the procedure. when swab stick was tried to put in her nose she resisted and started shouting. Guardians were explained about the urgency and importance of samples and were told to help in holding the patient along with the hospital attendants. After holding the patient's limbs, swab stick was again tried to put inside the nostril of the patient but as swab stick was inserted inside the nostril of the patient she moved her head due to which swab stick got broken inside her nostril from the indentation point. Then patient got more agitated. Psychiatrist was being called till that time FB was not touched and nursing staff tried to calm the patient. Psychiatrist came and advised intravenous anxiolytics and sedatives. After giving these drugs, FB was inspected by the ENT Resident and removed with the help of Tilley force. Nasal cavity was inspected for any trauma. Mild bleeding was present in the anterior nasal cavity. After achieving the hemostasis again NP sample was taken from the other nostril and OP sample was taken from the mouth. Xylometazoline drops were put inside the nasal cavities. swab samples were put inside the Universal Viral Transport media and was sealed using parafilm and sent to microbiology laboratory at 4-degree celsius. 


\section{Discussion}

Nasal foreign bodies are commonly encountered in the emergency department, particularly among children and mentally retarded patients [4]. As coronavirus disease 2019 (COVID-19) pandemic may be stressful for people. Fear and anxiety about a new disease and what could happen can be overwhelming and cause strong emotions in adults and children [5]. In the pandemic of COVID-19, large numbers of patients are tested with nasopharyngeal oropharyngeal swabs. Broken Swab stick within nasal cavity as retained foreign body is unusual but has been reported in recent months as a possible complication of sample testing in suspected patients. While giving samples patients sometimes become more anxious and irritable and do not cooperate with the health care worker who is taking NP and OP samples. Patient's doesn't allow health care workers to take sample and they resist and refuse to put swab stick into their oral and nasal cavity due to which swab stick breaks in their mouth or nose which leads to foreign body in the nose and oral cavity which is very unusual to see in nonpsychiatric patients. Here we will discuss how incidence of unusual nasal foreign body (swab stick) can be reduced in suspected covid 19 patients during sample taking. The development of new policies and procedures to address the several major areas requiring the engagement of different departments and staff workers to make the patient comfortable.

Clinical staff should actively and collaboratively engage the patient's caregivers in providing psychoeducation and other psychotherapeutic interventions to the patient to persuade resistant patients to take appropriate measures to prevent COVID-19. In cases of patient refusal for COVID-19 testing and resisting to give NP and OP samples, Attempts should be made to counsel the patient and encouraging, and motivating environment should be given to the patient to relieve his stress and anxiety. If still patient's stress not relieved, psychiatrist should be called to carefully assess and document the patient's capacity. Psychiatrist should counsel the patient. If a delay of testing is relatively safe, providing patients with psychiatric treatment that may enhance aspects of their capacity is appropriate. Anxiolytics, sedatives, or other antipsychotic drugs can be given after discussing the case with a psychiatrist depending upon the symptoms of the patient. Also, in order to access foreign body in an uncooperative patient, the question must be raised regarding whether or not a watch and wait strategy is more appropriate. If required restrictive measures must be considered as a last resort, employ methods that optimize safety for the patient and staff, and continuously re-evaluate restrictive measures while aiming to enlist patient assent and cooperation. Hospital ethics committees should play an important role in further developing and timely implementing these recommendations. As this will help in timely testing of covid 19 patients with decreased incidence of complications.

\section{Conclusion}

Coronavirus disease 2019 (COVID-19) is an infectious disease caused by severe acute respiratory syndrome coronavirus 2 (SARS-CoV-2). Over the past several months, infection has spread throughout the globe. During covid 19 pandemic, nasopharyngeal, and oropharyngeal samples are being taken of suspected patients by ENT department. Sometimes during sample taking patient's reluctant behaviour leads to breakage of swab stick from the indentation point inside the nasal or oral cavity which presents as an unusual foreign body in the nose or mouth. To avoid and reduce such complications we should counsel the patient to reduce their anxiety and stress. We should give them encouraging and motivating environment. Appropriate Anxiolytics, sedatives or other antipsychotic drugs can also be given. Psychiatric consultation should also be given to the patient.

\section{Summary}

New policies and procedures to address the several major areas requiring the engagement of different departments and staff workers to make the patient comfortable should be developed by the ethical committee of the hospital. Clinical staff should actively and collaboratively engage the patient caregivers in providing psychoeducation and other psychotherapeutic. This will help in timely testing of covid 19 patients with decreased incidence of complications.

\section{References}

1. Celenk F, Gokcen C, Celenk N, Baysal E, Durucu C, et al. (2013) Association between the self-insertion of nasal and aural foreign bodies and attention-deficit/hyperactivity disorder in children. Int J Pediatr Otorhinolaryngol7 7: 1291-1294.

2. https://www.who.int/dg/speeches/detail/who-director-general-sopening-remarks-at-the-media-briefing-on-covid-19-11-march-2020

3. https://www.cdc.gov/coronavirus/2019-ncov/lab/guidelines-clinicalspecimens

4. Werman HA (1987) Removal of foreign bodies of the nose. Emerg Med Clin North Am 5(2): 253-263.

5. https://www.cdc.gov/coronavirus/2019-ncov/daily-life-coping/ managing-stress-anxiety.html 
This work is licensed under Creative Commons Attribution 4.0 License

To Submit Your Article Click Here:

Submit Article

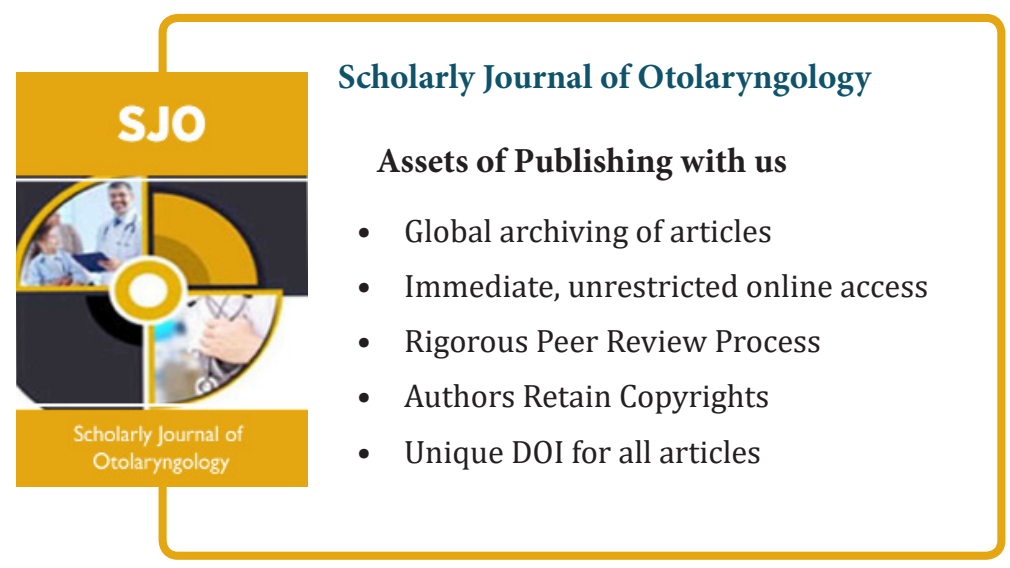

